\title{
Properties of some functionals associated with $h$-concave and quasilinear functions with applications to inequalities
}

\author{
Ludmila Nikolova' and Sanja Varošanec ${ }^{2 *}$
}

\section{*Correspondence: \\ varosans@math.hr \\ ${ }^{2}$ Department of Mathematics, \\ University of Zagreb, Zagreb, Croatia \\ Full list of author information is \\ available at the end of the article}

\begin{abstract}
We consider quasilinearity of the functional $(h \circ v) \cdot\left(\Phi \circ \frac{g}{v}\right)$, where $\Phi$ is a monotone $h$-concave ( $h$-convex) function, $v$ and $g$ are functionals with certain super(sub)additivity properties. Those general results are applied to some special functionals generated with several inequalities such as the Jensen, Jensen-Mercer, Beckenbach, Chebyshev and Milne inequalities.
\end{abstract}

Keywords: quasilinearity; $h$-concave function; monotone function; the Jensen functional; the Chebyshev functional

\section{Introduction and preliminaries}

In [1-4] Dragomir researched functionals which arise from quasilinear functionals related to the classical inequalities. For example, he considered the functionals $v \log \left(\frac{g}{v}\right)$ (in [1]), $v^{q-\frac{q}{p}} g^{q}, \frac{v^{q-\frac{q}{p}}}{g^{q}}$ (both in [2]), $v^{\frac{p-q}{p}} g^{q}$ (in [3]), and finally, $v \cdot\left(\Phi \circ \frac{g}{v}\right)$ (in [4]), where $v$ is additive, $g$ is super(sub)additive, $\Phi$ is a concave (convex) function and $p$ and $q$ are real numbers with some properties. In each paper he applied the given results about a composite functional to some of the classical inequalities such as: the Jensen, Hölder or Minkowski inequalities.

In this paper we generalize his results. We investigate similar functionals related to an $h$-convex function $\Phi$ under assumptions, which are weaker than the assumptions in the above-mentioned papers. In this introductory section, we give definitions and basic properties of several classes of functions.

Let $C$ be a convex cone in the linear space $X$ over $F(F=\mathbf{R}$ or $\mathbf{C})$, namely:

(a) $x, y \in C$ imply $x+y \in C$;

(b) $x \in C, \alpha>0$ imply $\alpha x \in C$.

Let $L$ be a real number, $L \neq 0$. A functional $f: C \rightarrow \mathbf{R}$ is called $L$-superadditive ( $L$-subadditive) on $C$ if

$$
f(x+y) \geq(\leq) L(f(x)+f(y))
$$

for any $x, y \in C$.

If $L=1$, then a functional $f$ is simply called superadditive (subadditive).

O2014 Nikolova and Varošanec; licensee Springer. This is an Open Access article distributed under the terms of the Creative Commons Attribution License (http://creativecommons.org/licenses/by/2.0), which permits unrestricted use, distribution, and reproduction in any medium, provided the original work is properly cited. 
Let $K$ be a real non-negative function. We say that a functional $f$ is $K$-positive homogeneous if

$$
f(t x)=K(t) f(x)
$$

for any $t \geq 0$ and $x \in C$.

In particular, if $K(t)=t^{k}$, then we simply say that $f$ is positive homogeneous on $C$ of order $k$. If $k=1$, we call it positive homogeneous.

It is easy to see that $K(1)=1$ and $K$ is multiplicative. Moreover, we have either $K \equiv 1$ or $K(0)=0$.

A function $h: J \subseteq \mathbf{R} \rightarrow \mathbf{R}$ is said to be a supermultiplicative function if

$$
h(x y) \geq h(x) h(y)
$$

for all $x, y \in J$.

If inequality (1) is reversed, then $h$ is said to be a submultiplicative function. If equality holds in (1), then $h$ is said to be a multiplicative function.

Example 1 The function $x \mapsto x^{s}$ is

1. superadditive and $L$-subadditive with $L=2^{s-1} \geq 1$ for $s \in(1, \infty)$,

2. subadditive and $L$-superadditive with $L=2^{s-1} \leq 1$ for $s \in(0,1]$,

3. $L$-subadditive with $L=2^{s-1} \leq \frac{1}{2}$ for $s<0$.

A function $f:[0, b] \rightarrow \mathbf{R}$ is called starshaped if $f(\alpha x) \leq \alpha f(x)$ for any $\alpha \in[0,1], x \in[0, b]$. In the sequel $I$ and $J$ are intervals in $\mathbf{R},(0,1) \subseteq J$ and functions $h$ and $f$ are non-negative functions defined on $J$ and $I$, respectively.

Definition 2 [5] Let $h: J \rightarrow \mathbf{R}$ be a non-negative function, $h \neq \equiv 0$. We say that $f: I \rightarrow \mathbf{R}$ is an $h$-convex function, if $f$ is non-negative and for all $x, y \in I, \alpha \in(0,1)$ we have

$$
f(\alpha x+(1-\alpha) y) \leq h(\alpha) f(x)+h(1-\alpha) f(y) .
$$

If the inequality is conversed, then $f$ is called an $h$-concave function.

Some properties of the $h$-convex functions are given in papers [5] and [6]. It is evident that this concept of $h$-convexity generalizes the concepts of classical non-negative convexity (for $h(t)=t)$; $s$-convexity in the second sense (for $\left.h(t)=t^{s}, s \in(0,1)\right)[7,8] ; P$-functions (for $h(t)=1$ ) [9]; and Godunova-Levin functions (for $\left.h(t)=t^{-1}\right)$ [10].

Example 3 It is known (see [5]) that the function $f(x)=x^{\lambda}$ is $s$-convex in the second sense if

$$
(\lambda \in(-\infty, 0] \cup[1, \infty) \text { and } s \leq 1) \text { or }(\lambda \in(0,1) \text { and } s \leq \lambda) .
$$

The function $f(x)=x^{\lambda}$ is $s$-concave in the second sense if

$$
(\lambda \in(0,1), s \geq 1) \text { or }(\lambda>1, s \geq \lambda) .
$$

Proposition 4 Let $0<s \leq 1$ and $f: I \rightarrow \mathbf{R}$ be s-convex, $0 \in I, f(0) \leq 0$. Then $f(\alpha x) \leq$ $\alpha^{s} f(x), 0 \leq \alpha \leq 1$, and $f$ is $2^{s-1}$-superadditive. 
Proof From s-convexity we have

$$
f(\alpha x+(1-\alpha) 0) \leq \alpha^{s} f(x)+(1-\alpha)^{s} f(0) \leq \alpha^{s} f(x) .
$$

The recently proved property and the property of the function $x \mapsto x^{s}$ give

$$
\begin{aligned}
f(a)+f(b) & =f\left((a+b) \frac{a}{a+b}\right)+f\left((a+b) \frac{b}{a+b}\right) \\
& \leq\left[\left(\frac{a}{a+b}\right)^{s}+\left(\frac{b}{a+b}\right)^{s}\right] f(a+b) \leq 2^{1-s} f(a+b) .
\end{aligned}
$$

If $s=1$, we get that $f$ is starshaped and hence superadditive.

Here we extract some properties proved in [5], which we use in this paper. Firstly, we give a result which allows us to use weights $\alpha, \beta$, the sum of which is not exactly 1 (as we have to use when we are working with convex functions) (see [5, Theorem 12]).

Theorem 5 Let I be an interval such that $0 \in I$.

(a) Let $f$ be h-convex on I and $f(0)=0$. If the function $h$ is supermultiplicative, then the inequality

$$
f(\alpha x+\beta y) \leq h(\alpha) f(x)+h(\beta) f(y)
$$

holds for all $x, y \in I$ and all $\alpha, \beta>0$ such that $\alpha+\beta \leq 1$.

(b) Let $f$ be h-concave on I. If the function $h$ is submultiplicative, then the inequality

$$
f(\alpha x+\beta y) \geq h(\alpha) f(x)+h(\beta) f(y)
$$

holds for all $x, y \in I$ and all $\alpha, \beta>0$ with $\alpha+\beta \leq 1$.

In fact, in the original paper [5] in part (b) we have assumption that $f(0)=0$ but a careful introspect of the proof gives us that non-negativity of the function $f$ is a sufficient assumption.

The next proposition gives us a tool for generating new $h$-convex functions.

Proposition 6 Let I be an interval such that $0 \in I$, and let $f$ be a non-negative function on $I$.

(a) Let $f$ be convex on I and $f(0)=0$. If $f$ is non-increasing and $s \in[1, \infty)$, then $f\left(x^{s}\right)$ is s-convex.

If $f$ is non-increasing, $s \in(0,1]$, then $f\left(x^{s}\right)$ is h-convex with $h(t)=2^{s-1} t^{s}$.

(b) Let $f$ be concave on I. If $f$ is non-decreasing and $s \in[1, \infty)$, then $f\left(x^{s}\right)$ is s-concave.

If $f$ is non-decreasing, $s \in(0,1]$, then $f\left(x^{s}\right)$ is h-concave with $h(t)=2^{s-1} t^{s}$.

Proof Let us prove the first part of case (b). The function $x \mapsto x^{s}$ is superadditive for $s \in$ $[1, \infty)$, so we have $(\alpha x+(1-\alpha) y)^{s} \geq \alpha^{s} x^{s}+(1-\alpha)^{s} y^{s}$. Since $f$ is non-decreasing, we obtain

$$
f\left((\alpha x+(1-\alpha) y)^{s}\right) \geq f\left(\alpha^{s} x^{s}+(1-\alpha)^{s} y^{s}\right) .
$$


Using superadditivity again, we have $\alpha^{s}+(1-\alpha)^{s} \leq 1$, and by the previous theorem we get

$$
f\left((\alpha x+(1-\alpha) y)^{s}\right) \geq \alpha^{s} f\left(x^{s}\right)+(1-\alpha)^{s} f\left(y^{s}\right) .
$$

Let us prove the last part of case (b). The function $x \mapsto x^{s}$ is $2^{s-1}$-superadditive for $s \in(0,1]$, so we have $(\alpha+(1-\alpha))^{s} \geq 2^{s-1}\left(\alpha^{s}+(1-\alpha)^{s}\right)$, i.e., $1 \geq 2^{s-1} \alpha^{s}+2^{s-1}(1-\alpha)^{s}$. So, these are two weights whose sum is less than or equal to 1 , and applying Theorem 5 to the concave function $f$ with arguments $x^{s}$ and $y^{s}$, we get

$$
\begin{aligned}
f\left(2^{s-1} \alpha^{s} x^{s}+2^{s-1}(1-\alpha)^{s} y^{s}\right) & \geq 2^{s-1} \alpha^{s} f\left(x^{s}\right)+2^{s-1}(1-\alpha)^{s} f\left(y^{s}\right) \\
& =h(\alpha) f\left(x^{s}\right)+h(1-\alpha) f\left(y^{s}\right) .
\end{aligned}
$$

Using $2^{s-1}$-superadditivity again, we obtain

$$
(\alpha x+(1-\alpha) y)^{s} \geq 2^{s-1}\left[(\alpha x)^{s}+((1-\alpha) y)^{s}\right] .
$$

Since $f$ is non-decreasing, and using previous results, we have

$$
\begin{aligned}
f\left((\alpha x+(1-\alpha) y)^{s}\right) & \geq f\left(2^{s-1} \alpha^{s} x^{s}+2^{s-1}(1-\alpha)^{s} y^{s}\right) \\
& \geq h(\alpha) f\left(x^{s}\right)+h(1-\alpha) f\left(y^{s}\right),
\end{aligned}
$$

which means that $f\left(x^{s}\right)$ is an $h$-concave function.

The other cases can be proved similarly.

Example 7 (i) The functions $f(x)=\arctan x, f(x)=\tanh x, f(x)=\frac{x}{1+x}$ are concave nondecreasing functions. By the previous proposition, the functions $f(x)=\arctan \left(x^{s}\right), f(x)=$ $\tanh \left(x^{s}\right), f(x)=\frac{x^{s}}{1+x^{s}}$, where $s>1$, are non-decreasing and $s$-concave, but not concave. Also, if $s \in(0,1]$, these functions are $h$-concave with $h(t)=2^{s-1} t^{s}$.

(ii) Another way to generate new $s$-convex ( $s$-concave) functions is using of the following statements: If $f$ is non-negative convex, then $f^{s}(x), s \in(0,1]$ is $s$-convex. If $f$ is non-negative concave, $s \in[1, \infty)$, then $f^{s}(x)$ is $s$-concave.

(iii) Consider the function

$$
f(x)= \begin{cases}x^{s}, & x \in[0,1] ; \\ x, & x \in(1, b],\end{cases}
$$

where $s>1$. This function is non-decreasing, convex on $(0,1]$, and starshaped on $[0, b]$, $b \geq 1$. Here we will show that this function is $s$-concave on $[0, b], b \geq 1$.

Let $x, y \in[0,1]$. Then $\alpha x+(1-\alpha) y \in[0,1]$ and

$$
\begin{aligned}
f(\alpha x+(1-\alpha) y) & =(\alpha x+(1-\alpha) y)^{s} \\
& \geq \alpha^{s} x^{s}+(1-\alpha)^{s} y^{s}=\alpha^{s} f(x)+(1-\alpha)^{s} f(y) .
\end{aligned}
$$

Let $x, y \in[1, b]$. Then $\alpha x+(1-\alpha) y \in[1, b]$ and

$$
\begin{aligned}
f(\alpha x+(1-\alpha) y) & =\alpha x+(1-\alpha) y \\
& \geq \alpha^{s} x+(1-\alpha)^{s} y=\alpha^{s} f(x)+(1-\alpha)^{s} f(y) .
\end{aligned}
$$


Let $x \in[0,1], y \in[1, b]$ and $\alpha x+(1-\alpha) y \in[1, b]$. Then

$$
\begin{aligned}
f(\alpha x+(1-\alpha) y) & =\alpha x+(1-\alpha) y \\
& \geq \alpha^{s} x^{s}+(1-\alpha)^{s} y=\alpha^{s} f(x)+(1-\alpha)^{s} f(y) .
\end{aligned}
$$

Let $x \in[0,1], y \in[1, b]$ and $\alpha x+(1-\alpha) y \in[0,1]$. Then we have

$$
\begin{aligned}
f(\alpha x+(1-\alpha) y) & =(\alpha x+(1-\alpha) y)^{s} \geq \alpha^{s} x^{s}+(1-\alpha)^{s} y^{s} \\
& \geq \alpha^{s} x^{s}+(1-\alpha)^{s} y=\alpha^{s} f(x)+(1-\alpha)^{s} f(y)
\end{aligned}
$$

since $y \geq 1, s \geq 1$. After considering all possibilities, we conclude that $f$ is $s$-concave.

The paper is arranged as follows. In the following section we prove some results about the functional $(h \circ v) \cdot\left(\Phi \circ \frac{g}{v}\right)$, where $\Phi$ is a monotone $h$-convex (h-concave) function. In the third section some applications of those results to the Jensen, Jensen-Mercer and Beckenbach functionals are given. Specific assumption in the third section is that the function $v$ is additive. The fourth section is devoted to some new superadditive mappings such as the Chebyshev and Milne functionals. A characteristic assumption in that section is that $v$ is superadditive.

\section{Functionals associated with monotone $\boldsymbol{h}$-concave and $\boldsymbol{h}$-convex functions}

Lemma 8 Let $x, y \in C$ and $f: C \rightarrow \mathbf{R}$ be a non-negative, L-superadditive and $K$-positive homogeneous functional on C. If $M \geq m>0$ are such that $x-m y$ and $M y-x \in C$, then

$$
\frac{1}{L} K(M) f(y) \geq f(x) \geq L K(m) f(y) .
$$

Proof Using $L$-superadditivity and $K$-positive homogeneity of $f$, we have

$$
\begin{aligned}
f(x) & =f(x-m y+m y) \geq L(f(x-m y)+f(m y)) \\
& \geq L f(m y)=L K(m) f(y)
\end{aligned}
$$

giving the second inequality. Similarly, we get the first inequality. Namely, using homogeneity and $L$-superadditivity, we get

$$
\frac{1}{L} K(M) f(y)=\frac{1}{L} f(M y)=\frac{1}{L} f(M y-x+x) \geq f(M y-x)+f(x) \geq f(x) .
$$

The above-proved lemma is a generalization of a result from [1] in which $f$ is superadditive and positive homogeneous of order $s$.

Theorem 9 Let $h$ be a non-negative function which is $k_{1}$-positive homogeneous. Let $C$ be a convex cone in the linear space $X$, and let $v: C \rightarrow(0, \infty)$ be an L-superadditive functional on $C$.

(i) If h is submultiplicative, $g: C \rightarrow[0, \infty)$ is an L-superadditive (L-subadditive) functional on $C$ and $\Phi:[0, \infty) \rightarrow[0, \infty)$ is h-concave and non-decreasing 
(non-increasing), then the functional $\eta_{\Phi}: C \rightarrow \mathbf{R}$ defined by

$$
\eta_{\Phi}(x):=h(v(x)) \Phi\left(\frac{g(x)}{v(x)}\right)
$$

is $k_{1}(L)$-superadditive on $C$.

(ii) If $h$ is supermultiplicative, $g$ is L-subadditive, $\Phi$ is $h$-convex and non-decreasing with $\Phi(0)=0$, then $\eta_{\Phi}$ is $k_{1}(L)$-subadditive.

Proof (i) Let us suppose that $h$ is submultiplicative, $g$ is $L$-superadditive, $\Phi$ is $h$-concave and non-decreasing. Let $\alpha=L \frac{v(x)}{v(x+y)}, \beta=L \frac{v(y)}{v(x+y)}$. Since $v$ is $L$-superadditive, we have $\alpha+\beta \leq$ 1 and

$$
\begin{aligned}
\Phi\left(\frac{g(x+y)}{v(x+y)}\right) & \geq \Phi\left(\frac{L g(x)+L g(y)}{v(x+y)}\right) \\
& =\Phi\left(\frac{L v(x)}{v(x+y)} \frac{g(x)}{v(x)}+\frac{L v(y)}{v(x+y)} \frac{g(y)}{v(y)}\right) \\
& \geq h\left(\frac{L v(x)}{v(x+y)}\right) \Phi\left(\frac{g(x)}{v(x)}\right)+h\left(\frac{L v(y)}{v(x+y)}\right) \Phi\left(\frac{g(y)}{v(y)}\right) \\
& =k_{1}(L)\left[h\left(\frac{v(x)}{v(x+y)}\right) \Phi\left(\frac{g(x)}{v(x)}\right)+h\left(\frac{v(y)}{v(x+y)}\right) \Phi\left(\frac{g(y)}{v(y)}\right)\right] \\
& \geq k_{1}(L)\left[\frac{h(v(x))}{h(v(x+y))} \Phi\left(\frac{g(x)}{v(x)}\right)+\frac{h(v(y))}{h(v(x+y))} \Phi\left(\frac{g(y)}{v(y)}\right)\right] .
\end{aligned}
$$

The first inequality holds because $\Phi$ is non-decreasing and $L$-superadditivity of $g$. The second inequality follows from Theorem 5 and $h$-concavity of $\Phi$. Next we use $k_{1}$-positive homogeneity of $h$ and finally the submultiplicativity of $h$. Multiplying with $h(v(x+y))$, we have

$$
h(v(x+y)) \Phi\left(\frac{g(x+y)}{v(x+y)}\right) \geq k_{1}(L)\left[h(v(x)) \Phi\left(\frac{g(x)}{v(x)}\right)+h(v(y)) \Phi\left(\frac{g(y)}{v(y)}\right)\right] .
$$

Hence $\eta_{\Phi}$ is $k_{1}(L)$-superadditive. The proofs of the other cases follow in a similar manner.

A superadditive and non-negative functional has the following boundedness property.

Corollary 10 Let $h$ be a non-negative submultiplicative function which is $k_{1}$-positive homogeneous. Let $C$ be a convex cone in the linear space $X$, and let $v: C \rightarrow(0, \infty)$ be Lsuperadditive and $k_{2}$-positive homogeneous on $C$. Let $x, y \in C$ and assume that there exist $M \geq m>0$ such that $x-m y$ and $M y-x \in C$. Let $K(t)=k_{1}\left(k_{2}(t)\right)$.

If $g: C \rightarrow[0, \infty)$ is an L-superadditive (L-subadditive) and $k_{2}$-positive homogeneous functional on $C$ and $\Phi:[0, \infty) \rightarrow[0, \infty)$ is h-concave and non-decreasing (non-increasing), then

$$
\frac{1}{k_{1}(L)} K(M) \eta_{\Phi}(y) \geq \eta_{\Phi}(x) \geq k_{1}(L) K(m) \eta_{\Phi}(y)
$$


Proof Note that $h(v(\alpha x))=h\left(k_{2}(\alpha) v(x)\right)=k_{1}\left(k_{2}(\alpha)\right) h(v(x))=K(\alpha) h(v(x))$. We observe that if $v$ and $g$ are $k_{2}$-positive homogeneous functionals, then $\eta_{\Phi}(x)=h(v(x)) \Phi\left(\frac{g(x)}{v(x)}\right)$ is a $K$-positive homogeneous functional and, by Theorem 9 , it follows that $\eta_{\Phi}$ is a $k_{1}(L)$ superadditive functional on $C$. By applying Lemma 8 we get the result.

Corollary 11 Let $h$ be a non-negative submultiplicative function which is positive homogeneous of order $s_{1}$. Let $C$ be a convex cone in the linear space $X$ and $v: C \rightarrow[0, \infty)$ be $L$-superadditive and positive homogeneous of order $s_{2}$ on $C$. Let $x, y \in C$ and assume that there exist $M \geq m>0$ such that $x-m y$ and $M y-x \in C$.

If $g: C \rightarrow[0, \infty)$ is an L-superadditive and positive homogeneous functional of order $s_{2}$ on $C$ and $\Phi:[0, \infty) \rightarrow[0, \infty)$ is h-concave and non-decreasing, then

$$
\frac{M^{s}}{L^{s_{1}}} \eta_{\Phi}(y) \geq \eta_{\Phi}(x) \geq m^{s} L^{s_{1}} \eta_{\Phi}(y)
$$

where $s=s_{1} s_{2}$.

Proof Put in the previous corollary $k_{1}(t)=t^{s_{1}}, k_{2}(t)=t^{s_{2}}$, and $K(t)=t^{s_{1} s_{2}}=t^{s}$.

Remark 12 If $L=1$, then the assumption about homogeneity of $h$ can be omitted and the statement of Theorem 9 still holds, namely we get superadditivity (subadditivity) of $\eta_{\Phi}$.

If we consider the additive function $v$, then using the same proof $(L=1$ and the first inequality is just equality), we get the following statements:

(i) If $g$ is superadditive (subadditive), $\Phi$ is $h$-concave and non-decreasing (non-increasing), where $h$ is submultiplicative, then the functional $\eta_{\Phi}$ is superadditive.

(ii) If $g$ is superadditive (subadditive), $\Phi$ is $h$-convex and non-increasing (non-decreasing), where $h$ is supermultiplicative, then the functional $\eta_{\Phi}$ is subadditive.

Comparing these statements with the results of Theorem 5 from paper [4], we see that if $\Phi$ is a non-negative function, then we have results for a wider class of functions $\Phi$, i.e., for $h$-concave or $h$-convex functions.

The case $s_{1}=1, h(t)=t$ gives results for concave $\Phi$, as it is in [4], but for $v$ and $g$ superadditive and $s_{2}$-positive homogeneous. The case when $v$ is only superadditive is important for applications - see the application to the Chebyshev and Milne functionals.

Moreover, Corollary 10 under assumptions that $v$ is additive and $L=1, k_{1}(t)=k_{2}(t)=t$, becomes the same as Corollary 1(a) from [4].

More about Corollary 10: If $h(t)=t^{s}, s_{2}=1, L=1$ and we use as an example $\Phi(x)=$ $\Phi_{1}^{s}(x), s \geq 1, \Phi_{1}$ is concave non-decreasing, then we get the result of Corollary 1 from [4]. However, we can also use the functions from Example 7 to get new results.

\section{Case 1: function $v$ is additive}

\section{Applications to Jensen-type inequalities}

Let $f$ be a real mapping on a convex subset $C_{1}$ of a linear space. Let us fix $n \in \mathbf{N}$ and $x_{i} \in C_{1}$ $(i=1, \ldots, n)$, and let $S_{+}(n):=\left\{\bar{p}=\left(p_{1}, \ldots, p_{n}\right): p_{i} \geq 0, i=1, \ldots, n\right.$ and $\left.P_{n}=\sum_{i=1}^{n} p_{i}>0\right\} . S_{+}(n)$ is a convex cone. 
As usual, the Jensen functional $J: S_{+}(n) \rightarrow \mathbf{R}$ is given by

$$
J(\bar{p}):=\sum_{i=1}^{n} p_{i} f\left(x_{i}\right)-P_{n} f\left(\frac{1}{P_{n}} \sum_{i=1}^{n} p_{i} x_{i}\right) .
$$

In fact, $J$ is a difference between the right-hand and the left-hand sides of the Jensen inequality for the convex function and it is a topic of investigation in many papers, see, for example, [11-13] and the references therein. It is known that $J$ is positive homogeneous; if $f$ is convex, then $J$ is non-negative and superadditive, while if $f$ is concave, then $J$ is nonpositive and subadditive. The boundedness property for the Jensen functional is proved in [1], i.e., if $M \geq m>0$ such that $M \bar{p} \geq \bar{q} \geq m \bar{p}$ (i.e., $M p_{i} \geq q_{i} \geq m p_{i}$ for each $i=1, \ldots, n$ ), then we get the property

$$
M J(\bar{p}) \geq J(\bar{q}) \geq m J(\bar{p})
$$

As an application of the results from the previous section, we have the following theorem.

Theorem 13 Let $h$ be a non-negative submultiplicative function and $f$ be convex. Let $\Phi$ be h-concave and non-decreasing on $[0, \infty)$. Then the composite functional $\eta_{\Phi}: S_{+}(n) \rightarrow R$ defined by

$$
\eta_{\Phi}(\bar{p})=h\left(P_{n}\right) \Phi\left(\sum_{i=1}^{n} \frac{p_{i}}{P_{n}} f\left(x_{i}\right)-f\left(\sum_{i=1}^{n} \frac{p_{i}}{P_{n}} x_{i}\right)\right)
$$

is superadditive.

Let, furthermore, $h$ be k-positive homogeneous. Let $\bar{p}, \bar{q} \in S_{+}(n)$ and let $M \geq m>0$ be such that $M \bar{p} \geq \bar{q} \geq m \bar{p}$. Then

$$
\begin{aligned}
k(M) h\left(P_{n}\right) \Phi\left(\frac{J(\bar{p})}{P_{n}}\right) & \geq h\left(Q_{n}\right) \Phi\left(\frac{J(\bar{q})}{Q_{n}}\right) \\
& \geq k(m) h\left(P_{n}\right) \Phi\left(\frac{J(\bar{p})}{P_{n}}\right) .
\end{aligned}
$$

Proof Take $v(\bar{p})=P_{n}$ and $g(\bar{p})=J(\bar{p})$. The functionals $v$ and $g$ are positive homogeneous, $v$ is additive and $g$ is superadditive. Using Theorem 9 we get that the composite functional $\eta_{\Phi}$ is superadditive on $S_{+}(n)$ and $k$-positive homogeneous. Hence, we apply Lemma 8 and get the wanted inequalities.

Remark 14 If $h(t)=t$, then we get results from [4]. In the same paper Dragomir discussed applications involving the Hölder and Minkowski functionals, but we leave that direction of investigation at this moment. In the rest of this section, we want to point out applications to some other Jensen-type functionals.

\section{On the Jensen-Steffensen conditions}

Now, let $f$ be a real function on an interval $I \subseteq \mathbf{R}$. In the previous theorem weights $p_{i}$ are non-negative and considered cone $C$ is the cone $S_{+}(n)$. For some choices of points 
$x_{1}, \ldots, x_{n} \in I$, this cone can be substituted with a larger cone. Let $\bar{x}=\left(x_{1}, \ldots, x_{n}\right)$ be fixed monotonic $n$-tuple of elements from $I$, and let us define the set $S(\bar{x}, n)$ by

$$
S(\bar{x}, n)=\left\{\bar{p} \in \mathbf{R}^{n}: P_{n}>0,0 \leq P_{k} \leq P_{n} \text {, where } P_{k}=\sum_{i=1}^{k} p_{i}, \sum_{i=1}^{n} \frac{p_{i}}{P_{n}} x_{i} \in I\right\} .
$$

The set $S(\bar{x}, n)$ is a cone. By the Jensen-Steffensen inequality [14, p.57], the difference $J(\bar{p})=$ $\sum_{i=1}^{n} p_{i} f\left(x_{i}\right)-P_{n} f\left(\sum_{i=1}^{n} \frac{p_{i}}{P_{n}} x_{i}\right)$, where $f$ is convex on $I$, is non-negative for each $\bar{p} \in S(\bar{x}, n)$. Using a similar proof as for the Jensen functional on $S_{+}(n)$, we get that $J$ is superadditive for a convex function $f$ and applying Theorem 9 we obtain that the functional $\eta_{\Phi}$ given by (4) is superadditive and the corresponding boundedness property holds.

The boundedness property of the Jensen functional under the Jensen-Steffensen conditions with an additional normalizing property $P_{n}=1$ was proved in [11] by using a different method.

\section{Applications to the Jensen-Mercer functional}

Mercer in paper [15] proved the Jensen-type inequality which includes boundary points of an interval. Namely, he stated the following result, which is nowadays called the JensenMercer inequality.

The Jensen-Mercer inequality. Let $\bar{p}=\left(p_{1}, \ldots, p_{n}\right)$ be a non-negative $n$-tuple with $P_{n}=$ $\sum_{i=1}^{n} p_{i}>0$, and let $x_{1}, x_{2}, \ldots, x_{n} \in[a, b]$. If the real function $f$ is convex on $[a, b]$, then

$$
f\left(a+b-\frac{1}{P_{n}} \sum_{i=1}^{n} p_{i} x_{i}\right) \leq f(a)+f(b)-\frac{1}{P_{n}} \sum_{i=1}^{n} p_{i} f\left(x_{i}\right) .
$$

If $f$ is concave, then the reversed inequality holds.

Let us fix $f:[a, b] \rightarrow \mathbf{R}$, and points $x_{i} \in[a, b], i=1,2, \ldots, n$. Let us define the JensenMercer functional as $J M: S_{+}(n) \rightarrow \mathbf{R}$

$$
J M(\bar{p})=P_{n}(f(a)+f(b))-\sum p_{i} f\left(x_{i}\right)-P_{n} f\left(a+b-\frac{1}{P_{n}} \sum p_{1} x_{i}\right) .
$$

It is easy to see that the functional $J M$ is positive homogeneous, non-negative for a convex function $f$ and non-positive for a concave function $f$. Superadditivity of the functional $J M$ was considered in [16]. In fact we have the following result.

Theorem 15 [16] Iff is convex, then the Jensen-Mercer functional JM is superadditive on $S_{+}(n)$.

Iff is concave, then JM is subadditive on $S_{+}(n)$.

Applying results of the second section, we have the following theorem.

Theorem 16 Let $h$ be a non-negative submultiplicative function, $f$ be a convex function, and let $\Phi:[0, \infty) \rightarrow[0, \infty)$ be an h-concave non-decreasing function. Then the functional $\zeta: S_{+}(n) \rightarrow \mathbf{R}$ defined by

$$
\zeta(\bar{p})=h\left(P_{n}\right) \Phi\left(f(a)+f(b)-\frac{1}{P_{n}} \sum_{i=1}^{n} p_{i} f\left(x_{i}\right)-f\left(a+b-\frac{1}{P_{n}} \sum_{i=1}^{n} p_{i} x_{i}\right)\right)
$$

is superadditive on $S_{+}(n)$. 
Proof Consider the functionals $v(\bar{p})=P_{n}$ and $g(\bar{p})=J M(\bar{p})$. The functional $v$ is additive and $g$ is superadditive and

$$
\eta_{\Phi}(\bar{p})=h(v(\bar{p})) \Phi\left(\frac{g(\bar{p})}{v(\bar{p})}\right)=\zeta(\bar{p}) .
$$

Hence, by applying Theorem 9, we get the desired result.

Corollary 17 Let us suppose that the assumptions of Theorem 16 are fulfilled, and let $h$ be $k$-positive homogeneous. If $\bar{p}, \bar{q} \in S_{+}(n)$ and $M \geq m>0$ are such that $M \bar{p} \geq \bar{q} \geq m \bar{p}$, then

$$
\begin{aligned}
k(M) h\left(P_{n}\right) \Phi\left(\frac{J M(\bar{p})}{P_{n}}\right) & \geq h\left(Q_{n}\right) \Phi\left(\frac{J M(\bar{q})}{Q_{n}}\right) \\
& \geq k(m) h\left(P_{n}\right) \Phi\left(\frac{J M(\bar{p})}{P_{n}}\right) .
\end{aligned}
$$

The proof follows from Corollary 10.

\section{Applications to the Beckenbach functional}

As [17, Theorem 5] shows, if $f$ is convex for $x \in[0, a]$ and starshaped in $[0, b], b>a$, then, for $x_{i} \in[0, b]$ and $\alpha_{i} \in(0,1), \sum_{i=1}^{n} \alpha_{i}=1$, we have

$$
f\left(\frac{a}{b} \sum_{i=1}^{n} \alpha_{i} x_{i}\right) \leq \frac{a}{b} \sum_{i=1}^{n} \alpha_{i} f\left(x_{i}\right) .
$$

That inequality is known as the Beckenbach inequality. Let us consider the Beckenbach functional $J_{a, b}$

$$
J_{a, b}(\bar{p})=\frac{a}{b} \sum_{i=1}^{n} p_{i} f\left(x_{i}\right)-P_{n} f\left(\frac{a}{b} \sum_{i=1}^{n} \frac{p_{i}}{P_{n}} x_{i}\right),
$$

where $\bar{p}, \bar{q} \in S_{+}(n)$ and $a, b, f$ satisfy assumptions of the Beckenbach inequality.

The above-mentioned theorem shows that $J_{a, b}(\bar{p}) \geq 0$.

Proposition 18 The functional $J_{a, b}$ is superadditive.

Proof It yields that

$$
\begin{aligned}
J_{a, b}(\bar{p} & +\bar{q})-J_{a, b}(\bar{p})-J_{a, b}(\bar{q}) \\
= & -\left(P_{n}+O_{n}\right) f\left(\frac{P_{n}}{P_{n}+Q_{n}} \frac{a}{b} \sum_{i=1}^{n} \frac{p_{i}}{P_{n}} x_{i}+\frac{Q_{n}}{P_{n}+Q_{n}} \frac{a}{b} \sum_{i=1}^{n} \frac{q_{i}}{Q_{n}} x_{i}\right) \\
& +P_{n} f\left(\frac{a}{b} \sum_{i=1}^{n} \frac{p_{i}}{P_{n}} x_{i}\right)+P_{n} f\left(\frac{a}{b} \sum_{i=1}^{n} \frac{q_{i}}{Q_{n}} x_{i}\right) \\
\geq & -\left(P_{n}+Q_{n}\right)\left[\frac{P_{n}}{P_{n}+Q_{n}} f\left(\frac{a}{b} \sum_{i=1}^{n} \frac{p_{i}}{P_{n}} x_{i}\right)+\frac{Q_{n}}{P_{n}+Q_{n}} f\left(\frac{a}{b} \sum_{i=1}^{n} \frac{q_{i}}{Q_{n}} x_{i}\right)\right] \\
& +P_{n} f\left(\frac{a}{b} \sum_{i=1}^{n} \frac{p_{i}}{P_{n}} x_{i}\right)+P_{n} f\left(\frac{a}{b} \sum_{i=1}^{n} \frac{q_{i}}{Q_{n}} x_{i}\right)=0,
\end{aligned}
$$


because $f$ is convex on $[0, a]$ and

$$
\frac{a}{b} \sum_{i=1}^{n} \frac{p_{i}}{P_{n}} x_{i} \leq a, \quad \frac{a}{b} \sum_{i=1}^{n} \frac{q_{i}}{Q_{n}} x_{i} \leq a .
$$

Theorem 19 Let $h$ be a non-negative submultiplicative function, let $f$ be a convex function, and let $\Phi:[0, \infty) \rightarrow[0, \infty)$ be an h-concave non-decreasing function. Then the functional $\eta_{\Phi}: S_{+}(n) \rightarrow \mathbf{R}$ defined by

$$
\eta_{\Phi}(\bar{p})=h\left(P_{n}\right) \Phi\left(\frac{a}{b} \sum_{i=1}^{n} \frac{p_{i}}{P_{n}} f\left(x_{i}\right)-f\left(\frac{a}{b} \sum_{i=1}^{n} \frac{p_{i}}{P_{n}} x_{i}\right)\right)
$$

is superadditive on $S_{+}(n)$. Furthermore, if $h$ is k-positive homogeneous, $\bar{p}, \bar{q} \in S_{+}(n)$ and $M \geq m>0$ such that $M \bar{p} \geq \bar{q} \geq m \bar{p}$, then

$$
\begin{aligned}
k(M) h\left(P_{n}\right) \Phi\left(\frac{J_{a, b}(\bar{p})}{P_{n}}\right) & \geq h\left(Q_{n}\right) \Phi\left(\frac{J_{a, b}(\bar{q})}{Q_{n}}\right) \\
& \geq k(m) h\left(P_{n}\right) \Phi\left(\frac{J_{a, b}(\bar{p})}{P_{n}}\right) .
\end{aligned}
$$

Proof Consider the functionals $v(\bar{p})=P_{n}$ and $g(\bar{p})=J_{a, b}(\bar{p})$. The functional $v$ is additive and $g$ is superadditive, and applying Theorem 9 we get that $\eta_{\Phi}$ is superadditive. The boundedness property follows from Corollary 10.

\section{Case 2: function $v$ is superadditive}

In the previous section the function $v$ was additive. Now, we will show some examples of applications with a superadditive function $v$.

\section{Applications to the Chebyshev functional for sums}

Let $\bar{a}$ and $\bar{b}$ be two real $n$-tuples. We call them similarly ordered if

$$
\left(a_{i}-a_{j}\right)\left(b_{i}-b_{j}\right) \geq 0
$$

for any $i, j=1, \ldots, n$. If the above inequality is reversed, then $n$-tuples are called oppositely ordered.

Let us denote

$$
T(\bar{a}, \bar{b}, \bar{p})=\sum_{i=1}^{n} p_{i} \sum_{i=1}^{n} p_{i} a_{i} b_{i}-\sum_{i=1}^{n} p_{i} a_{i} \sum_{i=1}^{n} p_{i} b_{i} .
$$

The statement of the classical Chebyshev inequality is the following (see [14, pp.197-204]).

The Chebyshev inequality. Let $\bar{a}=\left(a_{1}, \ldots, a_{n}\right)$ and $\bar{b}=\left(b_{1}, \ldots, b_{n}\right)$ be two $n$-tuples of real numbers and $\bar{p}=\left(p_{1}, \ldots, p_{n}\right)$ be a non-negative $n$-tuple. If $\bar{a}$ and $\bar{b}$ are similarly ordered, then the Chebyshev inequality

$$
T(\bar{a}, \bar{b}, \bar{p}) \geq 0
$$

holds. If $\bar{a}$ and $\bar{b}$ are oppositely ordered, then the reversed inequality holds. 
In the following theorem, we consider a quasilinear property of the Chebyshev functional $\bar{p} \mapsto T(\bar{a}, \bar{b}, \bar{p})$.

Theorem 20 If $\bar{a}$ and $\bar{b}$ are similarly ordered real $n$-tuples, $\bar{p} \geq 0$, then the functional $T(\bar{a}, \bar{b}, \bar{p})$ is superadditive in the variable $\bar{p}$. If $\bar{a}$ and $\bar{b}$ are oppositely ordered real $n$-tuples, then the functional $T(\bar{a}, \bar{b}, \bar{p})$ is subadditive.

Proof Let us suppose that $\bar{a}$ and $\bar{b}$ are similarly ordered $n$-tuples, and let us consider a sum $T(\bar{a}, \bar{b}, \bar{p}+\bar{q})-T(\bar{a}, \bar{b}, \bar{p})-T(\bar{a}, \bar{b}, \bar{q})$. We have

$$
\begin{aligned}
& T(\bar{a}, \bar{b}, \bar{p}+\bar{q})-T(\bar{a}, \bar{b}, \bar{p})-T(\bar{a}, \bar{b}, \bar{q}) \\
& =\sum_{i=1}^{n} p_{i} \sum_{i=1}^{n} q_{i} a_{i} b_{i}+\sum_{i=1}^{n} q_{i} \sum_{i=1}^{n} p_{i} a_{i} b_{i}-\sum_{i=1}^{n} p_{i} a_{i} \sum_{i=1}^{n} q_{i} b_{i} \\
& \quad-\sum_{i=1}^{n} q_{i} a_{i} \sum_{i=1}^{n} p_{i} b_{i}=L_{n} .
\end{aligned}
$$

After simple calculation we get

$$
L_{n+1}=L_{n}+\sum_{j=1}^{n}\left(p_{n+1} q_{j}+q_{n+1} p_{j}\right)\left(a_{j}-a_{n+1}\right)\left(b_{j}-b_{n+1}\right) .
$$

Since $\bar{p}$ and $\bar{q}$ are non-negative and $\bar{a}$ and $\bar{b}$ are similarly ordered, we have

$$
L_{n+1} \geq L_{n} \geq L_{n-1} \geq \cdots \geq L_{1}=0
$$

which means that $T(\bar{a}, \bar{b}, \bar{p})$ is superadditive. If $\bar{a}$ and $\bar{b}$ are oppositely ordered, the proof is similar.

Let us apply results from the second section to the functional $T(\bar{a}, \bar{b}, \bar{p})$.

Theorem 21 Let $h$ be a non-negative submultiplicative function, and $\Phi:[0, \infty) \rightarrow[0, \infty)$ is h-concave and non-decreasing.

(i) If $\bar{a}$ and $\bar{b}$ are similarly ordered, then the functional $\eta_{\Phi}(\bar{p})=h\left(P_{n}^{2}\right) \Phi\left(\frac{T(\bar{a}, \bar{b}, \bar{p})}{P_{n}^{2}}\right)$ is superadditive on $S_{+}(n)$.

Furthermore, if $h$ is $k$-positive homogeneous, $\bar{p}, \bar{q} \in S_{+}(n)$ and $M \geq m>0$ are such that $M \bar{p} \geq \bar{q} \geq m \bar{p}$, then

$$
\begin{aligned}
k\left(M^{2}\right) h\left(P_{n}^{2}\right) \Phi\left(\frac{T(\bar{a}, \bar{b}, \bar{p})}{P_{n}^{2}}\right) & \geq h\left(Q_{n}^{2}\right) \Phi\left(\frac{T(\bar{a}, \bar{b}, \bar{q})}{Q_{n}^{2}}\right) \\
& \geq k\left(m^{2}\right) h\left(P_{n}^{2}\right) \Phi\left(\frac{T(\bar{a}, \bar{b}, \bar{p})}{P_{n}^{2}}\right) .
\end{aligned}
$$

(ii) If $\bar{a}$ and $\bar{b}$ are oppositely ordered, then the functional $\eta_{\Phi}(\bar{p})=h\left(P_{n}^{2}\right) \Phi\left(\frac{-T(\bar{a}, \bar{b}, \bar{p})}{P_{n}^{2}}\right)$ is superadditive on $S_{+}(n)$. If, additionally, the assumptions on $h, \bar{p}, \bar{q}, M$ and $m$ are satisfied as in case (i), then inequalities (5) hold with substitution $T \rightarrow-T$. 
Proof If $\bar{a}$ and $\bar{b}$ are similarly ordered, let us define $v$ and $g$ as $v(\bar{p})=P_{n}^{2}$ and $g(\bar{p})=T(\bar{a}, \bar{b}, \bar{p})$. These functionals are positive homogeneous of order 2 and superadditive. By Theorem 9 with $L=1$ we have that $\eta_{\Phi}$ is superadditive, and by Corollary 10 for the functional $\eta_{\Phi}$ we obtain inequality (5).

If $\bar{a}$ and $\bar{b}$ are oppositely ordered, then the functional $-T(\bar{a}, \bar{b}, \bar{p})$ is superadditive and non-negative, and we proceed as in the proof of case (i).

Remark 22 If $\Phi(x)=x$, i.e., $h(t)=t, k(t)=t$, and if $\bar{a}$ and $\bar{b}$ are similarly ordered $n$-tuples, then for $\bar{p}, \bar{q}$ such that $M \bar{p} \geq \bar{q} \geq m \bar{p}$, we get

$$
M^{2} T(\bar{a}, \bar{b}, \bar{p}) \geq T(\bar{a}, \bar{b}, \bar{q}) \geq m^{2} T(\bar{a}, \bar{b}, \bar{p})
$$

If $\bar{p} \geq \bar{q}$, i.e., $M=1$, then from the above inequalities we get the following property of monotonicity:

$$
T(\bar{a}, \bar{b}, \bar{p}) \geq T(\bar{a}, \bar{b}, \bar{q})
$$

If $\bar{a}$ and $\bar{b}$ are oppositely ordered, then the reversed inequalities in (6) and (7) hold.

Let us take $\bar{p}=\overline{p^{(n)}}=\left(p_{1}, p_{2}, \ldots, p_{n}\right), \overline{p^{(n-1)}}=\left(p_{1}, p_{2}, \ldots, p_{n-1}, 0\right), \overline{p^{(n-2)}}=\left(p_{1}, p_{2}, \ldots, p_{n-2}\right.$, $0,0) \cdots \overline{p^{(2)}}=\left(p_{1}, p_{2}, 0, \ldots, 0,0\right)$. Since $\overline{p^{(n)}} \geq \overline{p^{(n-1)}} \geq \cdots \geq \overline{p^{(2)}}$ we can use the above monotonicity to obtain the following result.

Corollary 23 If $\bar{a}$ and $\bar{b}$ are similarly ordered $n$-tuples and $\bar{p} \geq 0$, then

$$
T\left(\bar{a}, \bar{b}, \overline{p^{(n)}}\right) \geq T\left(\bar{a}, \bar{b}, \overline{p^{(n-1)}}\right) \geq T\left(\bar{a}, \bar{b}, \overline{p^{(n-2)}}\right) \geq \cdots \geq T\left(\bar{a}, \bar{b}, \overline{p^{(2)}}\right) \geq 0
$$

and

$$
T(\bar{a}, \bar{b}, \bar{p}) \geq \max _{1 \leq i<j \leq n}\left[\left(p_{i}+p_{j}\right)\left(p_{i} a_{i} b_{i}+p_{j} a_{j} b_{j}\right)-\left(p_{i} a_{i}+p_{j} a_{j}\right)\left(p_{i} b_{i}+p_{j} b_{j}\right)\right]
$$

If $\bar{a}$ and $\bar{b}$ are oppositely ordered, then the reversed inequalities in the above inequalities hold with substitution $\max \rightarrow \min$ in the second result.

\section{The Chebyshev functional for integrals}

Let $f, g$ be real functions on $I=[a, b]$. Let $S_{+}(I)$ be the cone of non-negative functions $p$ on $I$ such that $p, p f, p g$ and $p f g$ are integrable. Denote

$$
\begin{aligned}
T(f, g, p)= & \int_{a}^{b} p(x) d x \int_{a}^{b} p(x) f(x) g(x) d x \\
& -\int_{a}^{b} p(x) f(x) d x \int_{a}^{b} p(x) g(x) d x .
\end{aligned}
$$

The Chebyshev inequality for integrals states that $T(f, g, p) \geq 0$ when $f$ and $g$ are similarly ordered, i.e.,

$$
(f(x)-f(y))(g(x)-g(y)) \geq 0 .
$$


If $f$ and $g$ are oppositely ordered, then $T(f, g, p) \leq 0$. It is known that the following identity holds:

$$
T(f, g, p)=\frac{1}{2} \int_{a}^{b} \int_{a}^{b} p(x) p(y)(f(x)-f(y))(g(x)-g(y)) d x d y .
$$

Using that identity, we obtain that

$$
\begin{aligned}
& T(f, g, p+q)-T(f, g, p)-T(f, g, q) \\
& \quad=\frac{1}{2} \int_{a}^{b} \int_{a}^{b}[p(x) q(y)+q(x) p(y)](f(x)-f(y))(g(x)-g(y)) d x d y \geq 0
\end{aligned}
$$

when $f$ and $g$ are similarly ordered. So we have superadditivity of this functional $p \mapsto$ $T(f, g, p)$ on the cone $S_{+}(I)$. If $f$ and $g$ are oppositely ordered, then the functional $-T(f, g, p)$ is superadditive. Here we will show only how Corollary 10 can be applied to this situation.

Corollary 24 Let $h$ be a non-negative submultiplicative function, which is $k$-positive homogeneous and $\Phi:[0, \infty) \rightarrow[0, \infty)$ is h-concave and non-decreasing. Let $f$ and $g$ be similarly ordered. If $p, q \in S_{+}(I)$ such that $P=\int_{a}^{b} p(x) d x>0, Q=\int_{a}^{b} q(x) d x>0$ and $M \geq m>0$ are such that $M p(x) \geq q(x) \geq m p(x)$, then

$$
\begin{aligned}
k\left(M^{2}\right) h\left(P^{2}\right) \Phi\left(\frac{T(f, g, p)}{P^{2}}\right) & \geq Q^{2} \Phi\left(\frac{T(f, g, q)}{Q^{2}}\right) \\
& \geq k\left(m^{2}\right) h\left(P^{2}\right) \Phi\left(\frac{T(f, g, p)}{P^{2}}\right) .
\end{aligned}
$$

Proof Let the function $v$ be defined by $v(p)=\left(\int_{a}^{b} p(x) d x\right)^{2}$. It is superadditive and positive homogeneous of order $s_{2}=2$. The function $g$ will be the Chebyshev functional $T(f, g, p)$. It is also positive homogeneous of order $s_{2}=2$, superadditive and non-negative. By Corollary 10 for the functional $\eta_{\Phi}(p)=h(v(p)) \Phi\left(\frac{g(p)}{v(p)}\right)=h\left(P^{2}\right) \Phi\left(\frac{T(f, g, p)}{P^{2}}\right)$ with $L=1, K(t)=k\left(t^{2}\right)$, we obtain the wanted inequality.

Remark 25 If $\Phi(x)=x$, i.e., $h(t)=t, k(t)=t$, and if the functions $f$ and $g$ are similarly ordered, then for $p, q \in S_{+}(I)$ such that $M p(x) \geq q(x) \geq m p(x)$, we get

$$
M^{2} T(f, g, p) \geq T(f, g, q) \geq m^{2} T(f, g, p) .
$$

If $p(x) \geq q(x)$, i.e., $M=1$, then from the above inequalities we get the following property of monotonicity:

$$
T(f, g, p) \geq T(f, g, q)
$$

If $f$ and $g$ are oppositely ordered, then the reversed inequalities hold.

\section{Applications to the Milne functional}

Let us consider the Milne inequality (see [18, pp.61-62]): Let $a_{i}, b_{i}, i=1, \ldots, n$, be positive real numbers. Then

$$
\sum_{i=1}^{n}\left(a_{i}+b_{i}\right) \sum_{i=1}^{n} \frac{a_{i} b_{i}}{a_{i}+b_{i}} \leq \sum_{i=1}^{n} a_{i} \sum_{i=1}^{n} b_{i}
$$


It is easy to get a weighted version of the Milne inequality using substitutions

$$
a_{i} \rightarrow p_{i} a_{i}, \quad b_{i} \rightarrow p_{i} b_{i}
$$

where $p_{1}, \ldots, p_{n}$ are positive real numbers. Of course, it can be improved to non-negative weights.

Define the Milne functional as follows:

$$
J_{\mathrm{Mi}}(\bar{p})=\sum_{i=1}^{n} p_{i} a_{i} \sum_{i=1}^{n} p_{i} b_{i}-\sum_{i=1}^{n} p_{i}\left(a_{i}+b_{i}\right) \sum_{i=1}^{n} \frac{p_{i} a_{i} b_{i}}{a_{i}+b_{i}}
$$

The weighted Milne inequality means that $J_{\mathrm{Mi}}(\bar{p}) \geq 0$. Also, it is easy to see that $J_{\mathrm{Mi}}(\alpha \bar{p})=$ $\alpha^{2} J_{\mathrm{Mi}}(\bar{p})$, i.e., $J_{\mathrm{Mi}}$ is positive homogeneous of order 2 .

Theorem 26 The functional $J_{\mathrm{Mi}}(\bar{p})$ is superadditive on $S_{+}(n)$.

Proof It yields that

$$
\begin{aligned}
J_{\mathrm{Mi}}(\bar{p} & +\bar{q})-J_{\mathrm{Mi}}(\bar{p})-J_{\mathrm{Mi}}(\bar{q}) \\
= & \sum_{i=1}^{n} p_{i} a_{i} \sum_{i=1}^{n} q_{i} b_{i}+\sum_{i=1}^{n} q_{i} a_{i} \sum_{i=1}^{n} p_{i} b_{i} \\
& \quad-\left(\sum_{i=1}^{n} p_{i}\left(a_{i}+b_{i}\right) \sum_{i=1}^{n} \frac{q_{i} a_{i} b_{i}}{a_{i}+b_{i}}+\sum_{i=1}^{n} q_{i}\left(a_{i}+b_{i}\right) \sum_{i=1}^{n} \frac{p_{i} a_{i} b_{i}}{a_{i}+b_{i}}\right)=L_{n} .
\end{aligned}
$$

After some (not so short, but simple) calculations, we get

$$
\begin{aligned}
L_{n+1}-L_{n}= & p_{n}\left(a_{n} \sum_{i=1}^{n} q_{i} b_{i}+b_{n} \sum_{i=1}^{n} q_{i} a_{i}-\frac{a_{n} b_{n}}{a_{n}+b_{n}} \sum_{i=1}^{n} q_{i}\left(a_{i}+b_{i}\right)\right. \\
& \left.-\left(a_{n}+b_{n}\right) \sum_{i=1}^{n} \frac{q_{i} a_{i} b_{i}}{a_{i}+b_{i}}\right) \\
& +q_{n}\left(a_{n} \sum_{i=1}^{n} p_{i} b_{i}+b_{n} \sum_{i=1}^{n} p_{i} a_{i}-\frac{a_{n} b_{n}}{a_{n}+b_{n}} \sum_{i=1}^{n} p_{i}\left(a_{i}+b_{i}\right)\right. \\
& \left.-\left(a_{n}+b_{n}\right) \sum_{i=1}^{n} \frac{p_{i} a_{i} b_{i}}{a_{i}+b_{i}}\right) .
\end{aligned}
$$

The term in the first brackets, for instance, can be represented like

$$
\frac{1}{a_{n}+b_{n}} \sum_{i=1}^{n} \frac{q_{i}}{a_{i}+b_{i}}\left(a_{n} b_{i}-a_{i} b_{n}\right)^{2}
$$

So we have that

$$
L_{n+1} \geq L_{n} \geq L_{n-1} \geq \cdots \geq L_{1}=0
$$

which means that $J_{\mathrm{Mi}}$ is superadditive and the proof is complete. 
We can get results similar to those from the previous subsection. Let $v(\bar{p})=P_{n}^{2}$ and $g(\bar{p})=$ $J_{\mathrm{Mi}}(\bar{p})$. Then the functional $\eta_{\Phi}$ defined by

$$
\eta_{\Phi}(\bar{p})=h\left(P_{n}^{2}\right) \Phi\left(\frac{J_{\mathrm{Mi}}(\bar{p})}{P_{n}^{2}}\right)
$$

is superadditive and it has boundedness property which follows from Corollary 10. As in Remark 25, we have the following chain of inequalities.

Corollary 27 If $\bar{a}, \bar{b}, \bar{p} \geq 0$, then

$$
J_{\mathrm{Mi}}\left(\overline{p^{(n)}}\right) \geq J_{\mathrm{Mi}}\left(\overline{p^{(n-1)}}\right) \geq J_{\mathrm{Mi}}\left(\overline{p^{(n-2)}}\right) \geq \cdots \geq J_{\mathrm{Mi}}\left(\overline{p^{(2)}}\right) \geq 0
$$

and

$$
\begin{aligned}
J_{\mathrm{Mi}}(\bar{p}) \geq & \max _{1 \leq i<j \leq n}\left[\left(p_{i} a_{i}+p_{j} a_{j}\right)\left(p_{i} b_{i}+p_{j} b_{j}\right)\right. \\
& \left.-\left(p_{i}\left(a_{i}+b_{i}\right)+p_{j}\left(a_{j}+b_{j}\right)\right)\left(\frac{p_{i} a_{i} b_{i}}{a_{i}+b_{i}}+\frac{p_{j} a_{j} b_{j}}{a_{j}+b_{j}}\right)\right] .
\end{aligned}
$$

\section{Competing interests}

The authors declare that they have no competing interests.

\section{Authors' contributions}

Both authors conceived of the study and carried out the proofs together. Both authors read and approved the final manuscript.

\section{Author details}

${ }^{1}$ Department of Mathematics and Informatics, Sofia University, Sofia, Bulgaria. ${ }^{2}$ Department of Mathematics, University of Zagreb, Zagreb, Croatia.

\section{Acknowledgements}

The research of the first author was partially supported by the Sofia University SRF under contract No. 184/2013. The research of the second author was supported by the Ministry of Science, Education and Sports of the Republic of Croatia under grants 058-1170889-1050.

\section{Received: 11 October 2013 Accepted: 7 January 2014 Published: 24 Jan 2014}

\section{References}

1. Dragomir, SS: Inequalities for superadditive functionals with applications. Bull. Aust. Math. Soc. 77, 401-411 (2008)

2. Dragomir, SS: Quasilinearity of some composite functionals with applications. Bull. Aust. Math. Soc. 83, 108-121 (2011)

3. Dragomir, SS: The quasilinearity of a family of functionals in linear spaces with applications in to inequalities. Riv. Mat. Univ. Parma 4, 135-149 (2013)

4. Dragomir, SS: Quasilinearity of some functionals associated with monotonic convex functions. J. Inequal. Appl. 2012, 276 (2012)

5. Varošanec, S: On h-convexity. J. Math. Anal. Appl. 326, 303-311 (2007)

6. Bombardelli, M, Varošanec, S: Properties of $h$-convex functions related to the Hermite-Hadamard-Fejer inequalities. Comput. Math. Appl. 58, 1869-1877 (2009)

7. Breckner, WW: Stetigkeitsaussagen fur eine Klasse verallgemeinerter konvexer funktionen in topologischen linearen Raumen. Publ. Inst. Math. 23, 13-20 (1978)

8. Hudzik, H, Maligranda, L: Some remarks on s-convex functions. Aequ. Math. 48, 100-111 (1994)

9. Pearce, CEM, Rubinov, AM: P-Functions, quasi-convex functions and Hadamard-type inequalities. J. Math. Anal. Appl. 240, 92-104 (1999)

10. Godunova, EK, Levin, Vl: Neravenstva dlja funkcii širokoga klassa, soderžaščego vypuklye, monotonnye i nekotorye drugie vidy funkcii. In: Vyčislitel. Mat. i. Mt. Fiz. Mežvuzov. Sb. Nauč. Trudov., pp. 138-142. MGPI, Moscow (1985) (Russian)

11. Barić, J, Matić, M, Pečarić, J: On the bounds for the normalized Jensen functional and Jensen-Steffensen inequality. Math. Inequal. Appl. 12,413-432 (2009)

12. Dragomir, SS: Bounds for the normalized Jensen's functional. Bull. Aust. Math. Soc. 74, 471-478 (2006) 
13. Dragomir, SS, Pečarić, J, Persson, LE: Properties of some functionals related to Jensen's inequality. Acta Math. Hung. 70, 129-143 (1996)

14. Pečarić, JE, Proschan, F, Tong, YL: Convex Functions, Partial Orderings, and Statistical Applications. Academic Press, Boston (1992)

15. Mercer, AMcD: A variant of Jensen's inequality. J. Inequal. Pure Appl. Math. 4(4), Article ID 73 (2003)

16. Krnić, M, Lovričević, N, Pečarić, J: On some properties of Jensen-Mercer's functional. J. Math. Inequal. 6, 125-139 (2012)

17. Beckenbach, EF: Superadditivity inequalities. Pac. J. Math. 14, 421-438 (1964)

18. Hardy, GH, Littlewood, JE, Pólya, G: Inequalities. Cambridge University Press, Cambridge (1952)

10.1186/1029-242X-2014-30

Cite this article as: Nikolova and Varošanec: Properties of some functionals associated with $h$-concave and quasilinear functions with applications to inequalities. Journal of Inequalities and Applications 2014, 2014:30

Submit your manuscript to a SpringerOpen ${ }^{\circ}$ journal and benefit from:

- Convenient online submission

Rigorous peer review

- Immediate publication on acceptance

- Open access: articles freely available online

- High visibility within the field

- Retaining the copyright to your article 